\title{
NEGLIGIBILITY IN NONLOCALLY CONVEX SPACES ${ }^{1}$
}

\author{
CHARLES A. RILEY
}

\begin{abstract}
A negligibility theorem is established in a linear topological space without assuming the existence of a convex body or linearly bounded open set.
\end{abstract}

1. A set $A$ in a topological space $X$ is negligible if $X$ is homeomorphic to $X \backslash A$. Negligibility investigations in linear topological spaces include [1], [2], [3], [4], [6], and [7]. Using shrinkable neighborhoods of Ives [5] and Klee [8], we adapt methods of Bessaga and Klee to prove the following theorem.

(1.1) TheOREM. Suppose $\left(X, \tau_{1}\right)$ is a linear topological space admitting a linear topology $\tau_{2} \subset \tau_{1}$ such that $\left(X, \tau_{2}\right)$ is metrizable and incomplete, and that $K$ is $\tau_{2}$-compact, $U$ is $\tau_{2}$-open with $[0,1] K \subset U$. Then there is a $\tau_{1}$-homeomorphism $h: X \rightarrow X \backslash \dot{K}$ with $h \mid X \backslash U=I d$.

(1.2) Corollary. Suppose $\left(X, \tau_{1}\right)$ is a metrizable complete linear topological space, and $\tau_{2}$ is metrizable and strictly weaker than $\tau_{1}$. If $K, U$ are as in (1.1), the conclusion holds.

(1.3) COROLLARY. Let $M$ be the space of a.e. finite Lebesgue measurable functions, $S$ the simple functions, and $C$ the continuous functions, all on $[0,1]$. If $S \subset X \subsetneq M$ or $C \subset X \subsetneq M$, and if convergence in $X$ implies convergence in measure, then subsets of $X$ which are compact in $M$ (with the topology of convergence in measure) are negligible in $X$.

(1.4) Corollary. Suppose the hypotheses of (2.1) hold, except $U \in \tau_{1}$, $K$ is $\tau_{1}$-compact, and $\tau_{2}$ contains a linearly bounded set. Then the conclusion of the theorem holds.

It follows from (1.3) that $M$-compact sets are negligible in $L^{p}, p>0$. A case of Anderson's result on $\alpha$-spaces [1] follows from the theorem.

\footnotetext{
Received by the editors March 30, 1972 and, in revised form, November 27, 1972 and February 8, 1973.

AMS (MOS) subject classifications (1970). Primary 57A20; Secondary 46A15.

Key words and phrases. Negligibility, shrinkable set, star-shaped set, convergence in measure.

${ }^{1}$ This paper is part of the author's Ph.D. thesis which was done under the direction of Professor D. E. Sanderson.
}

(C) American Mathematical Society 1973 
An $\alpha$-space is an infinite dimensional linear topological space with a Schauder basis $\left\{b_{n}\right\}$, continuous coordinates, and an open neighborhood $U$ of 0 such that $b_{n} \notin U$ for each $n$. If $(X, \tau)$ is an $\alpha$-space, then $X$ may be regarded as a linear subspace of $s$. If $X \neq s$, then $\tau_{1} \mid X \subset \tau$, where $\tau_{1}$ is the topology of coordinatewise convergence, and $X$ is dense in $\left(s, \tau_{1}\right)$, since if $x \in s,\left(x_{1}, x_{2}, \cdots, x_{n}, 0,0, \cdots\right) \in X$, and $\rightarrow x$. Thus $\left(X, \tau_{1}\right)$ is incomplete, and the theorem applies to show $\tau_{1}$-compact sets negligible. If $X=s$, and $X$ is metrizable, again $\tau_{1} \subset \tau$ and $b_{n} \rightarrow O\left(\tau_{1}\right)$, but $b_{n} \rightarrow O(\tau)$ since $b_{n} \notin U$. By the open mapping theorem, $\left(X, \tau_{1}\right)$ is incomplete, and again $\tau_{1}$-compact sets are negligible in $(X, \tau)$.

If $U$ is a set in a linear topological space, and $p \in \operatorname{Int} U$, then $U$ is shrinkable at $p$ if $[0,1)(U-p)^{-} \subset \operatorname{Int}(U-p)$. Notice that each ray $r$ from $p$ meets $\mathrm{Bd} U$ at most once, and that $r \cap \bar{U}$ is closed and connected. If $U$ is shrinkable at $p$, the gauge functional $\gamma_{U}(x, p)$ is defined by $x=p+$ $\gamma_{U}(x, p)\left(\pi_{U}(x, p)-p\right)$ in case ray $p x$ meets $\mathrm{Bd} U$ in $\pi_{U}(x, p)$. If $\boldsymbol{p} \boldsymbol{x} \subset U$ or $x=p$, then $\gamma_{U}(x, p)=0$. Ives [5] has shown that $\gamma_{U}(x, p)$ is continuous in $x$. It follows that $\pi_{U}(x, p)$, as a function of $x$, is continuous on its domain. According to Klee [8], each Hausdorff linear topological space has a basis at 0 of open sets, shrinkable at 0 . A set $A$ in a linear space is star-shaped at $a \in A$ if $t x+(1-t) a \in A$ whenever $x \in A, t \in[0,1)$. In the following $[A]$ will denote the convex hull of $A$.

(1.5) Lemma. Suppose $X$ is a metrizable linear topological space and $K, W \subset X$ with $K$ compact, $W$ open and shrinkable at 0 . If $x \in X, k \in K$ and $r>0$, then $\{k+\lambda(x-k) \mid \lambda \geqq 0\} \subset K+r W$ if and only if $\{\lambda(x-k) \mid \lambda \geqq 0\} \subset W$.

Proof. Assume the first inclusion, and take $\lambda \geqq 0$. For each $n$, $k+n r(x-k) \in K+r W$, so that $k+n r(x-k)=k_{n}+r w_{n}$ with $k_{n} \in K$, $w_{n} \in W$, and $w_{n}=(1 / r)\left(k-k_{n}\right)+n(x-k) \in W .\left\{k_{n}\right\}$ has a convergent subsequence $\left\{k_{n_{l}}\right\}$. If $l$ is large,

$$
(\lambda+1) / n_{l}<1, \quad \text { and }\left((\lambda+1) / n_{l}\right)\left((1 / r)\left(k-k_{n_{l}}\right)+n_{l}(x-k)\right) \in W .
$$

Letting $l \rightarrow \infty,(\lambda+1)(x-k) \in \bar{W}$. Therefore $\lambda(x-k) \in W$. The converse is clear.

Proofs of the following lemmas are straightforward and omitted. Lemma (1.9) is due to Klee [8].

(1.6) Lemma. Lèt $Y$ be a linear subspace of a linear topological space $X, y \in Y$, and $U$ open, shrinkable at $y$. Then $U \cap Y$ is shrinkable at $y$ in $Y$.

(1.7) Lemma. Let $\left(X, \tau_{1}\right)$ be a linear topological space, and $\tau_{2}$ a weaker linear topology for $X$. If $U \in \tau_{2}$ is $\tau_{2}$-shrinkable at 0 , then $U$ is $\tau_{1}$-shrinkable at $0, \bar{U}^{\tau_{1}}=\bar{U}^{\tau_{2}}$, and $\mathrm{Bd}_{\tau_{1}} U=\mathrm{Bd}_{\tau_{2}} U$. 
(1.8) Lemma. If $X$ is a linear space, and $A, B \subset X$ with $A$ star-shaped at $x \in A$, then $C=\bigcup\{\lambda A+(1-\lambda) B \mid \lambda \in[0,1]\}$ is star-shaped at $x$.

(1.9) Lemma. If $U$ is open, shrinkable at 0 , and $K$ is compact, starshaped at $k \in K$, then $U+K$ is shrinkable at $k$. In particular, if $K$ is convex, $U+K$ is shrinkable at each point of $K$.

We now prove (1.1). Let $\tilde{X}$ be a linear metric completion of $\left(X, \tau_{2}\right)$, and $\left\{\tilde{W}_{n}\right\}$ a basis of open sets, shrinkable at 0 , with $\tilde{W}_{n}^{-} \subset W_{n-1} . U=$ $\tilde{U} \cap X$ for some open $\tilde{U}$. Using $[0,1] K \subset U$ and the compactness of $K$, we can find $\tilde{x} \in \tilde{X} \mid X$ such that $\bigcup\{\lambda \tilde{x}+(1-\lambda) K \mid \lambda \in[0,1]\} \subset \tilde{U}$. Let $y_{n} \rightarrow \tilde{x}$ with $y_{n} \in U$. Again the compactness of $K$ implies the existence of $n_{1}$ such that

$$
\bigcup\left\{\lambda\left[\tilde{x}, y_{n_{1}}\right]+(1-\lambda) K \mid \lambda \in[0,1]\right\} \subset \tilde{U} .
$$

Let $x_{1}=y_{n_{1}}$ and $\widetilde{K}_{1}=\bigcup\left\{\lambda\left[\tilde{x}, x_{1}\right]+(1-\lambda) K \mid \lambda \in[0,1]\right\}$, a compact subset of $\tilde{U}$. There exists $l_{1}$ such that $\left(\widetilde{K}_{1}+3 \tilde{W}_{l_{1}}\right)-\subset \tilde{U}$. Let

$$
\begin{array}{ll}
\tilde{A}_{1}=\left[\tilde{x}, x_{1}\right]+\tilde{W}_{l_{1}}, & A_{1}=\tilde{A}_{1} \cap X, \\
\tilde{B}_{1}=\left[\tilde{x}, x_{1}\right]+2 \tilde{W}_{l_{1}}, & B_{1}=\tilde{B}_{1} \cap X, \\
\tilde{C}_{1}=\tilde{K}_{1}+2 \tilde{W}_{l_{1}}, & C_{1}=\tilde{C}_{1} \cap X, \\
\tilde{D}_{1}=\tilde{K}_{1}+3 \tilde{W}_{l_{1}}, & D_{1}=\tilde{D}_{1} \cap X .
\end{array}
$$

By (1.8), (1.9), (1.6) and (1.7), $A_{1}, B_{1}, C_{1}$ and $D_{1}$ are $\tau_{1}$-shrinkable at $x_{1}$.

$$
\begin{aligned}
\overline{A_{1}} & =\tilde{A}_{1}^{-} \cap X=\left(\left[\tilde{x}, x_{1}\right]+\tilde{W}_{l_{1}}\right) \cap X \\
& =\left(\left[\tilde{x}, x_{1}\right]+\tilde{W}_{l_{1}}^{-}\right) \cap X \subset\left(\left[\tilde{x}, x_{1}\right]+2 \tilde{W}_{l_{1}}\right) \cap X=B_{1} .
\end{aligned}
$$

Similarly we get $\bar{A}_{1} \subset B_{1} \subset C_{1} \subset \bar{C}_{1} \subset D_{1}$. The statements $\left\{x_{1}+\lambda\left(x-x_{1}\right) \mid \lambda \geqq 0\right\}$ $\subset A_{1}, \subset B_{1}, \subset C_{1}, \subset D_{1}$ are equivalent by (1.5). Now we define $h_{1}: X \rightarrow X$. $h_{1} \mid \bar{A}_{1} \cup\left(X \backslash D_{1}\right)=$ Id. $h_{1}\left|\bar{B}_{1} \backslash A_{1}: \bar{B}_{1}\right| A_{1} \rightarrow \bar{C}_{1} \backslash A_{1}$ is defined as follows. If $y \in \bar{B}_{1} \backslash A_{1}$, then $\pi_{A_{1}}\left(y, x_{1}\right)$ is defined and, by the above remark, so are $\pi_{B_{1}}\left(y, x_{1}\right), \pi_{C_{1}}\left(y, x_{1}\right)$ and $\pi_{D_{1}}\left(y, x_{1}\right)$. If $r$ is a ray from $x_{1}$, intersecting $\mathrm{Bd} A_{1}, h_{1}$ maps $\left(\bar{B}_{1} \mid A_{1}\right) \cap r$ linearly onto $\left(\bar{C}_{1} \mid A_{1}\right) \cap r$. Thus,

$$
h_{1}(y)=\pi_{A_{1}}\left(y, x_{1}\right)+\frac{\gamma_{A_{1}}\left(y, x_{1}\right)-1}{\gamma_{A_{1}}\left(\pi_{B_{1}}\left(y, x_{1}\right), x_{1}\right)-1}\left(\pi_{C_{1}}\left(y, x_{1}\right)-\pi_{A_{1}}\left(y, x_{1}\right)\right),
$$

is a continuous function. $h_{1}\left|\bar{B}_{1}\right| A_{1}$ has an inverse of the same form, so it is a homeomorphism. Similarly, we define $h_{1}\left|\bar{D}_{1}\right| B_{1}: \bar{D}_{1}\left|B_{1} \rightarrow \bar{D}_{1}\right| C_{1} . h_{1}$ is then a homeomorphism. Another compactness argument shows there exists $n_{2}>n_{1}$ such that

$$
\left[\tilde{x}, y_{n_{2}}\right] \subset \tilde{A_{1}} \text { and } \bigcup\left\{\lambda\left[\tilde{x}, y_{n_{2}}\right]+(1-\lambda) K \mid \lambda \in[0,1]\right\} \subset \tilde{C}_{1} .
$$


Let $x_{2}=y_{n_{2}}, \quad \tilde{K}_{2}=\bigcup\left\{\lambda\left[\tilde{x}, x_{2}\right]+(1-\lambda) K \mid \lambda \in[0,1]\right\}$. Then $\left[\tilde{x}, x_{2}\right] \subset \tilde{A}_{1}$, $\tilde{K}_{2} \subset \tilde{C}_{1}$ and there exists $l_{2}>l_{1}$ such that $\left(\left[\tilde{x}, x_{2}\right]+2 \tilde{W}_{l_{2}}\right)^{-\subset} \subset \tilde{A}_{1}$, $\left(\widetilde{K}_{2}+3 \tilde{W}_{l_{2}}\right)-\subset \tilde{C}_{1}$. Let

$$
\begin{array}{ll}
\tilde{A}_{2}=\left[\tilde{x}, x_{2}\right]+\tilde{W}_{l_{2}}, & A_{2}=\tilde{A}_{2} \cap X, \\
\tilde{B}_{2}=\left[\tilde{x}, x_{2}\right]+2 \tilde{W}_{l_{2}}, & B_{2}=\tilde{B}_{2} \cap X, \\
\tilde{C}_{2}=\tilde{K}_{2}+2 \tilde{W}_{l_{2}}, & C_{2}=\tilde{C}_{2} \cap X, \\
\tilde{D}_{2}=\tilde{K}_{2}+3 \tilde{W}_{l_{2}}, & D_{2}=\tilde{D}_{2} \cap X .
\end{array}
$$

As before, $A_{2}, B_{2}, C_{2}, D_{2}$ are $\tau_{1}$-shrinkable at $x_{2}, \bar{A}_{2} \subset B_{2} \subset C_{2} \subset \bar{C}_{2} \subset D_{2}$, and the statements $\left\{x_{2}+\lambda\left(x-x_{2}\right) \mid \lambda \geqq 0\right\} \subset A_{2}, \subset B_{2}, \subset C_{2}, \subset D_{2}$ are equivalent. Also $\bar{B}_{2} \subset A_{1}$ and $\bar{D}_{2} \subset C_{1}$. Define $h_{2}: X \rightarrow X$ so that

$$
\begin{aligned}
& h_{2}\left|\bar{A}_{2} \cup\left(X \mid D_{2}\right)=\mathrm{Id}, \quad h_{2}\right| \bar{B}_{2} \backslash A_{2}: \bar{B}_{2} \mid A_{2} \rightarrow \bar{C}_{2} \backslash A_{2}, \\
& h_{2}\left|\bar{D}_{2} \backslash B_{2}: \bar{D}_{2}\right| B_{2} \rightarrow \bar{D}_{2} \mid C_{2} .
\end{aligned}
$$

Note $h_{2}|X| C_{1}=$ Id. Continue, obtaining sets

$$
\begin{aligned}
& D_{1} \supset C_{1} \supset B_{1} \supset A_{1} \\
& \cup \cup \\
& D_{2} \supset C_{2} \supset B_{2} \supset A_{2} \\
& \cup \cup \\
& D_{3} \supset C_{3} \supset B_{3} \supset A_{3}
\end{aligned}
$$

and homeomorphisms $h_{n}: X \rightarrow X$ with

$$
\begin{aligned}
h_{n} \mid \bar{A}_{n} \cup\left(X \backslash D_{n}\right) & =\mathrm{Id}, \\
h_{n}\left|\bar{B}_{n} \backslash A_{n}: \bar{B}_{n}\right| A_{n} & \rightarrow \bar{C}_{n} \mid A_{n}, \\
h_{n}\left|\bar{D}_{n}\right| B_{n}: \bar{D}_{n} \mid B_{n} & \rightarrow \bar{D}_{n} \mid C_{n}, \\
h_{n}|X| C_{n-1} & =\text { Id. }
\end{aligned}
$$

We claim $\bigcap \bar{A}_{n}=\bigcap \bar{B}_{n}=\varphi$. Since $\bar{A}_{n} \subset \bar{B}_{n} \subset A_{n-1} \subset B_{n-1}$, it is sufficient to show $\cap B_{n}=\varphi$. If $y \in \bigcap B_{n}$, then $y \in X$, and $y \in\left[\tilde{x}, x_{n}\right]+2 \tilde{W}_{l_{n}}$, so that $y=\tilde{x}$, a contradiction. Also $\cap \bar{C}_{n}=\bigcap \bar{D}_{n}=K$. Suppose $y \in \bigcap D_{n}$. Then $y \in X$ and $y \in \tilde{K}_{n}+3 \tilde{W}_{l_{n}}$, so that $y=\lambda \tilde{x}+(1-\lambda) k$. If $\lambda \neq 0, \tilde{x}=$ $(1 / \lambda)(y-(1-\lambda) k) \in X$, a contradiction. Therefore $y=k \in K$. Thus $K \subset$ $\bigcap \bar{C}_{n} \subset \bigcap \bar{D}_{n} \subset \bigcap D_{n} \subset K$, since $\bar{D}_{n} \subset C_{n-1} \subset D_{n-1}$. If we trace the motion of a point $x \in X$ under the successive homeomorphisms $h_{1}, h_{2}, \cdots$, we see $x \notin \bar{B}_{n}$ for some $n$, and $h_{n+k} \cdots h_{2} h_{1}|X| \bar{B}_{n}=h_{n} \cdots h_{2} h_{1} \mid X \backslash \bar{B}_{n}$. Thus 
we may define a homeomorphism $h$ on $X$ by $h(x)=\cdots h_{2} h_{1}(x)$. It is not hard to see $h$ is onto $X \backslash K$. Since $X \backslash U \subset X \backslash D_{1}$, evidently $h \mid X \backslash U=I d$.

Corollary (1.2) is proved by noting Id: $\left(X, \tau_{1}\right) \rightarrow\left(X, \tau_{2}\right)$ is not an open map, so the open mapping theorem implies $\tau_{2}$ is incomplete. For Corollary (1.3) note that $X$ with the topology of convergence in measure is incomplete, since it is dense and a proper subspace of $M$. Finally, we prove Corollary (1.4).

Using the hypothesis we can find $U_{1} \in \tau_{1}, V_{1} \in \tau_{2}$, both linearly bounded and $\tau_{1}$-shrinkable at zero, with $[0,1] K \subset U_{1} \subset U$ and $U_{1} \subset V_{1}$. Since $[0,1] K$ is $\tau_{1}$-compact, $[0,1] K \subset r U_{1}$ for some $r \in(0,1)$. There is a $\tau_{1^{-}}$ homeomorphism $j: X \rightarrow X$ such that

$$
j\left|r \bar{U}_{1} \cup\left(X \mid 2 V_{1}\right)=\mathrm{Id}, \quad j\right| \bar{U}_{1}\left|r U_{1}: \bar{U}_{1}\right| r U_{1} \rightarrow \bar{V}_{1} \mid r U_{1},
$$

and

$$
j\left|2 \bar{V}_{1}\right| U_{1}: 2 \bar{V}_{1}\left|U_{1} \rightarrow 2 \bar{V}_{1}\right| V_{1} .
$$

By the theorem, there exists a $\tau_{1}$-homeomorphism $h: X \rightarrow X \backslash K$ such that $h \mid X \backslash V_{1}=$ Id. Then $j^{-1} h j: X \rightarrow X \backslash K$ is a $\tau_{1}$-homeomorphism fixed on $X \backslash U$.

2. In the proof of (1.1), the homeomorphisms Id, $h_{1}, h_{2} h_{1}, h_{3} h_{2} h_{1}, \cdots$ may be regarded as successive stages of an isotopy whose final homeomorphism is $h: X \rightarrow X \backslash K$. There are obvious ways to fill the gaps, but the details are tedious. The statement of the isotopy theorem below is patterned after Klee's [6], and the corollary extends his theorem to an arbitrary normed linear space. A full development of these results will appear elsewhere.

(2.1) THEOREM. Suppose $\left(X, \tau_{1}\right)$ is a linear topological space admitting a metrizable incomplete linear topology $\tau_{2} \subset \tau_{1}$. If $U \in \tau_{2}$ and $K$ is $\tau_{2^{-}}$ compact with $[0,1] K \subset U$, then there exists a $\tau_{1}$-embedding $H: X \times[0,1] \rightarrow$ $X \times[0,1]$ such that if $f_{t}(x)=p_{1} H(x, t)$ (projection on the first coordinate) for $t \in[0,1]$, then $\left\{f_{t}\right\}$ has the following properties.

1. $f_{t}: X \rightarrow X$. is a $\tau_{1}$-homeomorphism for each $t \in[0,1)$.

2. $f_{0}=\mathrm{Id}$.

3. $f_{1}: X \rightarrow X \backslash K$ is a $\tau_{1}$-homeomorphism.

4. For each $t \in[0,1], f_{t} \mid X \backslash U=\mathrm{Id}$.

5. $\lim _{t \rightarrow 1} f_{1} f_{t}^{-1}=\operatorname{Id}_{X}\left(\tau_{1}\right)$ and $\lim _{t \rightarrow 1} f_{t} f_{1}^{-1}=\operatorname{Id}_{X \backslash K}\left(\tau_{1}\right)$ with the convergence uniform on each $\tau_{2}$-compact set.

(2.2) Corollary. Suppose the hypotheses of the theorem hold, except that $U \in \tau_{1}, K$ is $\tau_{1}$-compact and $[0,1] K \subset U$. Suppose also $\tau_{2}$ contains a linearly bounded set. Then the conclusions of the theorem hold (with limits uniform on $\tau_{1}$-compact sets). 


\section{REFERENCES}

1. R. D. Anderson, On a theorem of Klee, Proc. Amer. Math. Soc. 17 (1966), 14011404. MR 34 \#4864.

2. R. D. Anderson and R. H. Bing, A complete elementary proof that Hilbert space is homeomorphic to the countable infinite product of lines, Bull. Amer. Math. Soc. 74 (1968), 771-792. MR 37 \#5837.

3. C. Bessaga and V. L. Klee, Two topological properties of topological linear spaces, Israel J. Math. 2 (1964), 211-220. MR 31 \#5055.

4. C. Bessaga, Negligible sets in linear topological spaces, Bull. Acad. Polon. Sci. Sér. Sci. Math. Astronom. Phys. 16 (1968), 117-119. MR 37 \#1946.

5. R. T. Ives, Semi-convexity and locally bounded spaces, Ph.D. thesis, Univ. of Washington, Seattle, Wash., 1957.

6. V. L. Klee, Convex bodies and periodic homeomorphisms in Hilbert space, Trans. Amer. Math. Soc. 74 (1953), 10-43. MR 14, 989.

7. - A note on topological properties of normed linear spaces, Proc. Amer. Math. Soc. 7 (1956), 673-674. MR 17, 1227.

8. - Shrinkable neighborhoods in Hausdorff linear spaces, Math. Ann. 141 (1960), 281-285. MR 24 \#A1003.

Department of Mathematics, Iowa State University, Ames, Iowa 50010

Department of Mathematics, Keene State College, Keene, New Hampshire 03431 (Current address) 